\title{
Beneficios metabólicos de realizar ejercicio en estado de ayuno
}

\author{
Metabolic benefits of exercise \\ in the fasted state
}

\begin{abstract}
The high rate of obesity in sedentary population, coupled with the increase in diseases associated with this lifestyle, have led us to seek new strategies to enhance the physical training that will favor mobilization, uptake and utilization of fats and glucose. Doing exercise in the fasted state is one that has proved more promising. There is sufficient evidence on the benefits of physical activity during fasting producing a favorable hormonal environment, activation of mediators such as AMPK and LPL activation, among others. Performing aerobic exercise of moderate intensity in this environment favors fasting lipid oxidation, decreasing intramyocellular triglycerides, visceral fat and LDL cholesterol, compared with the benefits of exercise in the postprandial state. There is an important need for more studies of physical activity in the fasted state that include patients with chronic non-transmissible diseases and resistance training.

Key words: exercise, lipolysis, fasted state.
\end{abstract}

Astrid von Oetinger G. $(1,2)$

Luz María Trujillo G. $(2,3)$ Univias de la Salud Universidad San Sebastián, Santiago, Chile. (2) Escuela de Kinesiología, Facultad de Odontología y Salud, Universidad Diego Portales, Santiago, Chile. (3) Centro Tratamiento del Sueño LINDE S.A. Santiago, Chile.

$$
\begin{array}{r}
\text { Dirigir la correspondencia a: } \\
\text { Klga. } \\
\text { Luz María Trujillo Gittermann, } \\
\text { Escuela Kinesiología, } \\
\text { Facultad de Salud y Odontología, } \\
\text { Universidad Diego Portales, } \\
\text { Ejército 219, Santiago. } \\
\text { Teléfono: 56-226762256 } \\
\text { E-mail: luzmariatrujillo.aga@gmail.com }
\end{array}
$$

Este trabajo fue recibido el 25 de Noviembre de 2014 y aceptado para ser publicado el 20 de Mayo de 2015.

\section{INTRODUCCIÓN}

El mundo sufre una nueva epidemia, la obesidad, que deja claro las paradojas de un desarrollo poco equitativo. Mientras las hambrunas azotan a partes del mundo, en otras la población aumenta de manera preocupante su peso corporal; ésta es la Ilamada carga del siglo XXI. Según la Organización Mundial de la Salud (OMS), esta epidemia afecta a nivel mundial a más de mil seiscientos millones de personas adultas con sobrepeso, de las cuales al menos cuatrocientos millones de adultos son clínicamente obesos. La "globesidad", como ha sido también llamada, no respeta divisiones entre países desarrollados y subdesarrollados y en estos últimos muchas veces convive con problemas de desnutrición $(1,2)$.

El mayor consumo de calorías que las necesarias debido a alimentos pobres nutricionalmente, pero ricos en grasas saturadas y carbohidratos, combinado con bajo nivel de actividad física, han elevado en el mundo al triple las cifras de obesidad desde 1980 a la fecha. Según la OMS, la obesidad y el sobrepeso plantean un alto riesgo de padecer enfermedades crónicas no transmisibles como son: la diabetes tipo 2, la hipertensión arterial, las enfermedades cardiovasculares, las coronarias y algunos tipos de cáncer (3-8).

Diferentes estudios han mostrado que el ejercicio de predominio aeróbico es un estímulo potente para mejorar la capacidad cardiovascular y prevenir la obesidad junto a sus enfermedades relacionadas, tales como hipertensión arterial, diabetes mellitus y dislipidemia. En el año 2007, el American College of Sport Medicine determinó que el ejercicio de predominio aeróbico, de intensidad moderada a intensa (40 - 60 $\%$ del consumo máximo de oxígeno), con una duración mayor a 30 minutos, por 5 días a la semana, es recomendable para la población de 18 a 65 años a fin de promover y mantener la salud evitando riesgo de patologías cardiovasculares $(9,10)$.

Generalmente, los individuos con enfermedades crónicas no transmisible realizan escasa actividad física, por lo que en los últimos años se han diseñado diferentes esquemas de ejercicio para potenciar la movilización, captación y utilización de grasas y de glucosa, con el fin de optimizar la efectividad del tratamiento no farmacológico y lograr mejores resultados a mediano plazo en estos pacientes (11-15).

Entre las prácticas que se han evaluado, la realización de ejercicio en estado de ayuno es una de las que ha resultado ser más promisoria $(16,17)$.

El objetivo del presente artículo fue revisar los antecedentes que evidencian y explican los beneficios de realizar ejercicio en ayuno en personas con patologías crónicas no transmisibles o bien con riesgo de padecerlas.

\section{DEFINICIÓN DEL AYUNO}

La definición de ayuno por parte del diccionario del idioma español Real Academia Española define al ayuno como:" abstinencia de toda comida y bebida desde las doce de la 
noche antecedente" (18).

Desde el punto de vista fisiológico, el ayuno se define como la situación metabólica que se circunscribe a la mañana posterior a una noche (10 -14 horas) sin comer (19).

\section{ADAPTACIÓN FISIOLÓGICA Y REGULACIÓN HORMONAL DEL METABOLISMO EN ESTADO DE AYUNO}

Las células en nuestro organismo obtienen energía para realizar su trabajo principalmente a partir de la oxidación de los nutrientes. Por lo tanto, las células requieren aporte de nutrientes que permitan la síntesis de Adenosin trifosfato, (ATP), para el óptimo funcionamiento de los procesos celulares; proceso que se logra gracias a la oxidación de la glucosa, de los ácidos grasos libres, los aminoácidos y los cuerpos cetónicos. Sin embargo, el ser humano se alimenta de manera intermitente, por lo que el aporte, la distribución y el uso de los nutrientes se modifican constantemente (20).

$\mathrm{Al}$ analizar las fases metabólicas del consumo energético, éstas se dividen en cuatro fases: la fase digestiva o absortiva (postprandial), que se produce durante las 2-3 horas que se tarda en digerir el alimento ingerido; la fase postabsortiva, que se produce entre las comidas; la fase de ayuno que corresponde al periodo entre la última ingesta antes de dormir y el desayuno; la fase de ejercicio agotador que requiere aporte energético importante durante la realización de éste (21).

Los procesos metabólicos en condición de ayuno son variables y dependen directamente de la duración de éste, con adaptaciones que buscan la conservación de la vida. Los mecanismos fisiológicos involucrados corresponden a la disminución del consumo de glucosa por parte del tejido muscular, adiposo y hepático provocándose una activación paralela de mecanismos que aumentan la producción de glucosa. Posterior a ello, otros nutrientes como son los ácidos grasos y cuerpos cetónicos comienzan a aumentar también su producción y utilización (19).

Entre las principales señales fisiológicas que provoca el ayuno tenemos: bioquímicas y hormonales. En cuanto a las primeras, una de las señales más importantes corresponde al aumento en el neuropéptido $Y$, considerado uno de los principales péptidos orexígenos; éste es liberado, a nivel periférico, de las neuronas simpáticas siendo capaz de inhibir la adenilato ciclasa vía proteína Gi (proteína G inhibitoria) principalmente en el tejido adiposo subcutáneo estimulando así la lipólisis $(22,23)$. Otra señal importante es la disminución de la glicemia, la que es significativa sólo después de 24 horas de ayuno. Cuando la glicemia alcanza valores menores a $75 \mathrm{mg} /$ $\mathrm{dL}$ gatilla un aumento significativo plasmático de hormonas contrareguladoras (Glucagón, noradrenalina, cortisol y hormona del crecimiento) y el péptido natriurético (AN) (19).Lo anterior sumado a la disminución de leptina e insulina ponen en marcha procesos metabólicos, como son la glucogenólisis, proteólisis, lipólisis, gluconeogénesis, cetogénesis y consumo energético reducido $(19,24)$.

Hay que destacar al tejido hepático como el principal regulador de la glicemia, sin embargo cuando el ayuno se prolonga el aparato renal colabora estimulando la gluconeogénesis $(25,26)$.

Durante el ayuno, condición en que los niveles de insulina están bajos, la captación de glucosa por parte del tejido muscular esquelético se reduce al mínimo; situación que privilegia el aporte de glucosa a los tejidos consumidores por excelencia: el sistema nervioso y glóbulos rojos. $(20,27)$. De esta manera los niveles bajos de insulina, presentes durante el ayuno, corresponde a un poderoso agente lipolítico, lo que favorece la utilización de ácidos grasos libres $(14,15)$.

Sumado a lo anterior es importante destacar el ritmo circadiano de las catecolaminas, que denotan niveles aumentados de estas durante las mañanas lo que provoca una disminución en la actividad de la lipoproteína lipasa y un aumento de la lipasa sensible a hormona (HLS) en el tejido adiposo, incrementando la movilización y utilización de ácidos grasos libres $(12,13)$. El resultado final de esta respuesta neuroendocrina determina una mayor proporción epinefrina/insulina plasmáticas $(13,14)$, creando un ambiente marcadamente lipolítico que sustenta la efectividad de realizar actividad física en esta condición.

\section{METABOLISMO DE LA FIBRA MUSCULAR ESQUELÉTICA EN AYUNO}

Durante el ayuno, la carencia de aporte externo limita la disponibilidad de glucosa, lo cual se compensa con un incremento en la oxidación de ácidos grasos en el músculo esquelético y en el resto de los tejidos, esto con el objeto de preservar la glicemia para suplir a los tejidos dependientes de glucosa (28). Las adaptaciones metabólicas en el músculo esquelético que ocurren en estado de ayuno, llevan a coordinar una respuesta transcripcional que promueve la utilización lipídica como sustrato energético por parte de las mitocondrias. En las modulaciones transcripcionales descritas, el coactivador PGC-1 alfa, (siglas en inglés; Peroxisome proliferator-activated receptor gamma coactivator) y la familia de factores de transcripción del tipo FOXOs (sigla en inglés, Forkhead box), parecen desempeñar un importante papel, ya que ambos se encuentran relacionados con la regulación de la biogénesis mitocondrial y con el control del metabolismo de ácidos grasos (29).

La actividad de PGC-1 alfa y FOXOs son influenciadas por el control de su nivel de acetilación a través de sirtuína-1 (SIRT-1), una proteína desacetilasa dependiente de Nicotinamida adenina dinucleótido, NAD+. Como resultado, el SIRT-1 actúa como un regulador maestro de las adaptaciones del músculo esquelético frente a las variaciones en disponibilidad de nutrientes (30). Sin embargo, la regulación de SIRT-1 aún se desconoce en su totalidad, destacando un estudio (31), que demuestra que la proteína quinasa activada por AMP (AMPK), enzima cuya actividad promueve la captación y utilización de glucosa y ácidos grasos libres plasmáticos por parte del músculo, actuaría como sensor primario de estrés energético, requiriéndose su actividad para iniciar las adaptaciones dependientes de SIRT-1(32).

Por su parte, la AMPK es activada por adenosin monofosfato, (AMP), un metabolito que aumenta en la medida que disminuye la carga energética de la célula, se considera que esta enzima juega un papel pivotal en la regulación de los flujos metabólicos dentro del músculo, actuando como un verdadero "sensor energético" y activando la oxidación de ácidos grasos y carbohidratos $(33,34)$. La reciente demostración de que la enzima posee una subunidad que une glicógeno y que esta unión lleva a la inhibición de la misma (35), sustenta la idea de que el glicógeno, aparte de su condición de reserva energética, es un regulador activo de la oxidación de hidratos de carbono y en mayor medida, de ácidos grasos. De esta manera, la disminución de glicógeno intramuscular, propio de estados de ayuno, así como de ejercicio de alta intensidad, provocaría la activación de la AMPK, favoreciendo el switch metabólico que conduce a una mayor oxidación de ácidos grasos (33-35).

Es por ello que la realización de una sesión de ejercicio en estado de ayuno, implica una situación donde el sujeto 
presenta una disminución significativa de sus niveles de glicógeno, liberando a la AMPK de su inhibición, lo que incrementa notablemente el consumo de grasas, ya que a la carencia del sustrato alternativo (glicógeno) se suma la estimulación del catabolismo lipídico por la activación de la AMPK (33-35).

\section{ROL DE LA LIPOPROTEÍNA LIPASA EN EL EJERCICIO EN AYUNO}

La hidrólisis de los triglicéridos (Quilomicrones, VLDL (siglas en inglés; very low density lipoprotein)) a ácidos grasos libres y glicerol comienza gracias a la acción de la lipoproteína lipasa, (LPL), enzima que se encuentra en el endotelio capilar de muchos tejidos como el adiposo, muscular esquelético y cardiaco principalmente. La LPL, es la enzima tasa limitante en la hidrólisis de triglicéridos (TG), por lo que es un importante regulador de las concentraciones de TG en plasma (36). Existen varios estudios que sugieren que la insulina genera en humanos cambios en la actividad de la LPL, tanto en la isoforma de tejido adiposo (a-LPL) como en la muscular (mLPL) $(36,37)$. Se ha demostrado en diferentes estudios que el ejercicio, aún en sesiones breves, regularía la actividad de la LPL y su expresión genética en el músculo esquelético (36-38). También se ha demostrado que luego de ingerir dietas pobres en carbohidratos, donde los niveles de insulina son bajos -al igual que los valores glicémicos- el nivel de actividad m-LPL es significativamente mayor a los observados con dietas altas en hidratos de carbono $(37,40)$. Además, hay que considerar la activación que produce el ejercicio físico de la LPL no ocurre hasta 4 horas post ejercicio y dura entre 18-24 horas $(36,41,42)$.

Se ha evidenciado que el estado de ayuno y el ejercicio generan aumento en la actividad en las distintas isoformas de la LPL. Ambas condiciones, ayuno más ejercicio, determinan bajos niveles de insulina, aumento en la oxidación de lípidos, a través de mayor estimulación de la $\mathrm{LPL}$, e incremento en los niveles plasmáticos de las hormonas contraregulatorias; logrando suplir los requerimientos energéticos que demanda el cuerpo en ejercicio (37-39).

\section{EJERCICIO AEROBICO Y METABOLISMO DE HIDRATOS DE CARBONO \\ Durante ayuno / postprandial}

El músculo esquelético cumple el rol más importante en el metabolismo de la glucosa, captando alrededor del 75\% de toda la glucosa luego de la estimulación insulínica. La limitación en la disponibilidad de carbohidratos ha demostrado ser beneficioso en aumentar el transporte de ácidos grasos tanto durante el ejercicio como posterior a éste (18).

Un estudio realizado por Van Proeyen $\mathrm{K}$. et al el año 2010, en jóvenes sanos, sometidos a entrenamiento físico en estado de ayuno o bien con aporte de dieta hipercalórica: obtuvieron que el entrenamiento físico en ayuno aumentó significativamente el contenido de Glut-4 en $28 \%$ y la fosforilación de la AMP-Kinasa en 25\%, traslocasa/CD36 en 30\% y la carnitin palmitoiltransferasa 1 mRNA (CPT1mRNA) en $30 \%$ comparado con el grupo control. Lo anterior indica que la tasa de oxidación de grasas, tolerancia a la glucosa y la sensibilidad a la insulina aumenta significativamente comparado con el grupo control (43).

Respecto a la ingesta de carbohidratos durante el ejercicio, es sabido que tiene un efecto ergogénico, retrasando el comienzo de la fatiga y mejorando el rendimiento deportivo de resistencia siempre y cuando tenga una duración mayor a una hora (44). Sin embargo, existe evidencia que realizar ejercicio en estado de depleción de carbohidratos puede ayudar a obtener adaptaciones metabólicas aumentando la capacidad oxidativa muscular y la degradación de lípidos intra miocelulares $(16,17,43)$.

Un estudio realizado el año 2008 por De Bock K. y cols., comparó a un grupo de hombres sometidos a entrenamiento físico en estado de ayuno y otro grupo con aporte de carbohidratos, obteniendo como resultado un aumento significativo de FATBPm (fatty acid binding protein in muscle) en el grupo entrenado en ayunas, lo que significa mayor cantidad de transportadores que son determinantes en la tasa de captación de ácidos grasos (45).

\section{EJERCICIO AERÓBICO Y METABOLISMO DE LOS ÁCIDOS GRASOS \\ Durante ayuno / postprandial}

En estado postprandiales los niveles de insulina aumentan, respecto a lo anterior hay que destacar su rol antilipolítico, el más importante en el ser humano. Ésta hormona es capaz de inactivar a la proteína kinasa $A$, disminuyendo la fosforilación de la lipasa hormono sensible (HSL), pudiendo llegar a afectar 95\% de la lipólisis $(46,47)$. Por el contrario en estados de ayuno sus niveles plasmáticos son bajos lo que favorece un ambiente fisiológico para la lipólisis.

Los ácidos grasos son muy importantes como sustratos energéticos para el músculo esquelético en contracción, principalmente durante el ejercicio prolongado (45). Diferentes fuentes de triacilglicerol contribuyen a la liberación de ácidos grasos durante el ejercicio físico, donde las principales fuentes son el tejido adiposo subcutáneo y el visceral, en menor medida las lipoproteínas circulantes y los depósitos locales en el músculo (48). Las catecolaminas son las principales responsables de que esta condición ocurra a través de la activación de los receptores adrenérgicos $\beta 1$ y $\beta 2$ y paralelamente a ello los NP representan la mayor señal no-adrenérgica lipolítica en el ser humano. Ellos activan la proteína G la que es capaz de fosforilar la HSL (49).

Achten J. et al (2004) demostraron que sujetos sometidos a entrenamiento físico, ya sea en situación de ayuno o con ingesta de hidratos de carbono (tanto antes como durante el ejercicio) la degradación de triglicéridos intramusculares, (IMTG), durante el estado de ayuna ocurre principalmente en las fibras tipo I .Concluyendo que ésta degradación se ve limitada en el estado de aporte de hidratos de carbono (48).

La acumulación de IMTG está asociada con la insulinoresistencia en la población sedentaria $(50,51)$. Se ha evidenciado que una dieta alta en grasa genera un desbalance entre los depósitos de grasas y su oxidación en la célula muscular, provocando una disminución de la sensibilidad insulínica $(52,53)$.

El estado de ayuna favorece el control lipídico postprandial, lo cual ha sido demostrado por Enevoldsen LH. et al (2004), quienes estudiaron a sujetos que realizaban ejercicio (una hora de bicicleta al 55\% VO2max), demostrando una disminución significativa en las concentraciones de VLDL y en la concentración de insulina plasmática, en comparación con la realización del mismo ejercicio una hora postprandial. Los autores concluyeron que realizar ejercicio en condiciones de ayuno provoca un predominio del estado lipolítico (39).

Asimismo Zang JQ. y cols. (1998) compararon el efecto de realizar 60 minutos de ejercicio, al $60 \%$ VO2max, en ayuna de 12 horas, con respecto a hacerlo en estado postprandial (ejercicio 1 hora post ingesta), concluyendo que realizar ejercicio en estado de ayuno provoca beneficios metabólicos respecto a los triglicéridos y el metabolismo del HDL (high 
density lipoprotein), lo que disminuye la progresión de los procesos ateroscleróticos (54).

\section{EJERCICIO EN ESTADO DE AYUNO}

A manera de resumen, los cambios endocrinos observados en ayuno son; el aumento del glucagón, catecolaminas y cortisol, así como el incremento de la hormona del crecimiento, favorecen un ambiente marcadamente lipolítico. A lo anterior se suma el aumento del AN quien cumple un importante rol en la lipólisis inducida por el ejercicio (55).

La leptina e insulina muestran un descenso en sus concentraciones, provocando una disminución del glicógeno muscular, esta condición endocrina además estimula el aumento de la $L P L$, potenciando a los mediadores del transporte de ácidos grasos, como lo son el FATBPm, CD36 y el CPT. Todos estos cambios, con aumento de la fosforilación de la AMPK, Ilevan a una mayor utilización de lípidos durante la realización de ejercicio en éste ambiente (figura 1).

\section{CONCLUSIONES}

La evidencia analizada respalda los beneficios de realizar ejercicio aeróbico de intensidad moderada a intensa en condición de ayuno, esto por distintos factores hormonales, enzimáticos y fisiológicos que resultan en una mayor oxidación de lípidos. Además de esto la evidencia respalda que la realización de actividad física en estado de supresión de carbohidratos favorece la tolerancia a la glucosa y la sensibilidad a la insulina, fundamental para pacientes que tienen síndrome metabólico.

Es importante recalcar que el entrenamiento y tipo de ejercicio a realizar debe ser acorde al objetivo que se busque, además hay sujetos que no toleran bien las actividades en ayuna, por lo que la planificación y objetivos del entrenamiento deben ser individualizados.

Faltan más estudios que muestren protocolos claros de actividad física en ayuna, donde también se puedan incluir los ejercicios resistidos y de intervalo de alta intensidad. Además la mayoría de la evidencia científica analizada se ha realizado en poblaciones sanas, es importante incluir en futuros estudios a pacientes con distintas patologías para tener más claridad sobre los beneficios que pueden tener distintos grupos de pacientes al realizar ejercicios en este ambiente.

\section{RESUMEN}

El alta tasa de obesidad y sedentarismo de la población, sumado al aumento de las patologías asociadas a éste estilo de vida, nos han llevado a buscar nuevas estrategias de entrenamiento físico que potencien la movilización, captación y utilización de grasas y glucosa. La realización de ejercicio en estado de ayuno es una de las que ha resultado ser más promisoria. Hay evidencia suficiente sobre los beneficios de realizar actividad física en ayunas, ya sea por el ambiente hormonal favorable, la activación de mediadores como el AMPK y la activación de la LPL, entre otros. La realización de ejercicio aeróbico de intensidad moderada en este ambiente de ayuno favorece la oxidación de lípidos, la disminución de los triglicéridos intramiocelulares, la grasa visceral y del colesterol LDL, en comparación con los beneficios del ejercicio realizado en ambiente postprandial. Existe una necesidad de un mayor número de estudios con actividad física en ayuna, donde se incluya a paciente con enfermedades crónicas no transmisibles

\section{FIGURA 1}

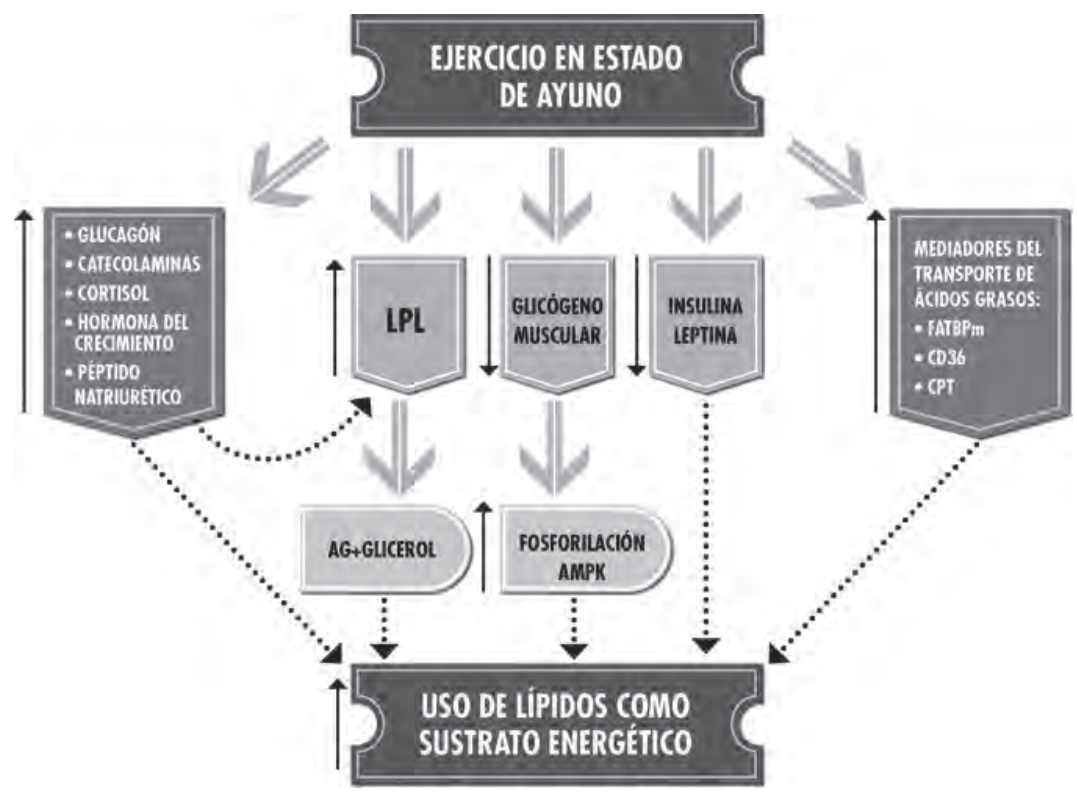

LPL: Lipoproteína Lipasa, AMPK: Proteína quinasa activada por AMP, FATBPm: fatty acid binding protein in muscle, CD36: Ácido Graso translocasa,

CPT: Carnitin palmitoiltransferasa. 
y los ejercicios de tipo resistido.

Palabras clave: ejercicio, lipolisis, estado de ayuno.

\section{BIBLIOGRAFÍA}

1. Organización Mundial de la Salud (OMS), Informe Macroeconomía y salud.2005. http://www.who.int/mediacentre/ factsheets/fs311/es/

2. Chopra M, Galbraith S, Darton-Hill I.A global response to a global problem: the epidemic of overnutrition. Bull World Health Organ.2002; 80:952-8.

3. Yach, D, Hawkes C, Gould CL \& Hofman KJ. The global burden of chronic diseases: overcoming impediments to prevention and control. JAMA. 2004; 291: 2616-22.

4. WHO. World Health Organization. The World Health Report 2002. Reducing Risks, Promoting Healthy Life. WHO 2002. Disponible en: http://www.who.int/whr/2002/en/ whr02_en.pdf [Consultado el 2 de Octubre de 2014].

5. MINSAL. Ministerio de Salud. Gobierno de Chile. Encuesta Nacional de Salud ENS Chile 2009-2010. Chile 2011. Disponible en: http://www.redsalud.gov.cl/portal/url/item/99 c12b89738d80d5e04001011e0113f8.pdf [Consultado el 2 de Octubre 2014].

6. World Health Organization. Definition, diagnosis and classification of diabetes mellitus and its complications .Part 1: Diagnosis and classification of diabetes mellitus. Report Who Consulation. 99.2 Geneva, Switzerland. 1990.

7. Valenzuela A. La Obesidad y sus comorbilidades. Primera edición 2002. Editorial Mediterráneo Ltda. 2002; 23 : 409-29.

8. Leitzmann MF, Park Y, Blair A ,Ballard-Barbash R, Mouw $T$, Hollenbeck AR. Physical activity recommendations and decreased risk of mortality. Arch Intern Med. 2007; 167:2453-60.

9. American College of Sports Medicine. Exercise prescription. In: ACSM's Guidelines for Exercise Testing and Prescription, Edited by Whaley MH. Baltimore, MD: Lippincott Williams \& Wilking, 7th Ed. 2006.

10. Haskell WL, Lee IM, Pate RR, Powell KE, Blair KE, Franklin BA, Macera CA, Heath GW, Thompson PD, Bauman A. Physical Activity and Public Health: Updated Recommendation for Adults From the American College of Sports Medicine and the American Heart Association. Circulation 2007;116:1081-93.

11. Stich V, De Glisezinski I, Berlan M, Bulow J, Galitzky J, Harant I, Suljkovicova H, Lafontan M, Riviere D, and Crampes F. Adipose tissue lipolysis is increased during a repeated bout of aerobic exercise. J Appl Physiol. 2000; 88:1277-83.

12. Goto K, Ishii N, Mizuno A, and Takamatsu K. Enhancement of fat metabolism by repeated bouts of moderate endurance exercise. J Appl Physiol.2007; 102: 2158 - 64.

13. Goto K, Ishii N, Sugihara S, Yosshioka T, Takamatsu K. Effects of resistance exercise on lipolysis during subsequent submaximal exercise. Med Sci Sports Exerc. 2007; 39: 308-15.

14. Ronsen O, Haug E, Pedersen BK, Bahr R. Increased neuroendocrine responses to repeated bout of endurance exercise. Med Sci Sports Exerc. 2001; 33: 568-75.

15. Ronsen $O$, Lea $T$, Bahr R, Pedersen BK. Enhanced plasma $I L-6$ and $I L-1$ responses to repeated vs. Single bouts of prolonged cycling in elite athletes. J Appl Physiol. 2002; 92: 2533-47.

16. Van Proyen K, Szlufcik K, Nielens H, Ramaekers M, Hespel $P$. Beneficial metabolic adaptations due to endurance exercise training in the fasted state. J Appl Physiol. 2011; 110: $236-45$.

17. De bock $K$, Richter EA, Russell AP, Eijnde BO, Derave W, Ramaekers M, Koninckx E, Leger B, Verhaeghe J, Hespel $P$. Exercise in the fasted state facilitates fibre type-specific intramyocellular lipid breakdown and stimulates glycogen resynthesis in humans. J Physiol. 2005: 564 (2): 649-60.

18. DeFronzo RA, Ferrannini E, Sato $Y$, Feling $P$, Wahren J. Synergistic interaction between exercise and insulin on peripheral glucose uptake. J Clin Invest. 1981; 68: 1468-74.

19. Albero R, Sanz A, Playán J. Metabolismo en el ayuno. Endocrinol Nutr. 2004;51(4):139-48.

20. Longo D V, Mattson PM. Fasting: Molecular Mechanisms and Clinical Applications. Cell Metab. 2014; 19: 181-92.

21. Koeppen BM and Stanton BA y Levy Fisiología. Sexta edición.Editorial Elsevier. 2009; 38: 664-95.

22. Serradeil-Le GC, Lafontan M, Raufaste D, Marchand $J$, Pouzet B, Casellas P, Pascal M, Maffrand JP, Le FG. Characterization of NPY receptors controlling lipolysis and leptin secretion in human adipocytes. FEBS Letters. 2000; 475: 150-6.

23. Castan I, Valet $P$, Larrouy $D$, Voisin $T$, Remaury A, Daviaud $D$, Laburthe $M$, Lafontan $M$. Distribution of PYY receptors in human fat cell: an antilipolytic system alongside the a2-adrenergic system. Am J Physiol. 1993; 265: E74-E80.

24. Bergendahl $M$, Vance $M L$, Iranmanesh $A$, Thorner $M O$, Veldhuis JD. Fasting as a metabolic stress paradigm selectively amplifies cortisol secretory burst mass and delays the time of maximal nyctohemeral cortisol concentrations in health men. J Clin Endocrinol Metab. 1996;81:692-9.

25. Klempel MC, Kroeger CM, Bhutani S, Trepanowski JF, Varady $K A$. Intermittent fasting combined with calorie restriction is effective for weight loss and cardio-protection in obese women. J Nutr. 2012; 11 (98): 2-9.

26. Harvie $M N$, Pegington $M$, Mattson $M P$, Frystyk J, Dillon $B$, Evans $G$, et al: The effects of intermittent or continuous energy restriction on weight loss and metabolic disease risk markers: a randomized trial in young overweight women. Int J Obes. (Lond) 2011; 35 (5):714-27.

27. Buschemeyer WC, Klink JC, Mavropoulos JC, Poulton SH, Demark-Wahnefried W, Hursting SD, et al: Effect of intermittent fasting with or without caloric restriction on prostate cancer growth and survival in SCID mice. Prostate. 2010; 70(10):1037-43.

28. Cahill GF, Herrera MG, Morgan AP, Soeldner JS, Steinke J, Levy PL, Reichard GA, Kipnis DM. Hormone-fuel interrelationships during fasting. J Clin Invest 1966; 45: 1751-69.

29. Gross DN, van den Heuvel AP, Birnbaum MJ. The role of FoxO in the regulation of metabolism. Oncogene. 2008; 27: 2320-36.

30. Fulco $M$, Cen $Y$, Zhao P, Hoffman EP, McBurney MW, Sauve AA, Sartorelli V. Glucose restriction inhibits skeletal myoblast differentiation by activating SIRT1 through AMPK-mediated regulation of Nampt. Dev Cell. 2008; 14: 661-73.

31. Canto $C$, Jiang L, Deshmukh A, Mataki CH, Coste A, Lagouge $M$, Zierath J, Interdependence of AMPK and SIRT1 for Metabolic Adaptation to Fasting and Exercise in Skeletal Muscle. Cell Metab. 2010; 11: 213-9.

32. Bergeron $R$, Previs SF. Effects of AICAR infusion in vivo on glucose and lipid metabolism on lean and obese Zucker rats. Diabetes 2002; 50:1076-82.

33. Goodyear, L.J. AMPK a critical signaling intermediary for exercise stimulated glucose transport. Exercise Sport Sc. 
Review. 2000; 28: 113-6.

34. Winder W. W. Energy-sensing and signaling by AMPK in skeletal muscle. J. Appl. Physiol. 2001; 91: 1017-28.

35. Steinberg G. and Bruce K. AMPK in Health and disease. Physiology Rev. 2009; 89: 1025-78.

36. Kiens B, Lithell $H$, Mikines KJ, Richter EA. Effects of insulin and exercise on muscle lipoprotein lipase activity in man and its relation to insulin action. J Clin Invest. 1989; 84:1124-9.

37. Jonida Haxhi, Alessandro Scotto di Palumbo,Massimo Sacchetti. Exercising for metabolic control: is timing important? Ann Nutr Metab. 2013; 62:14-25.

38. Herd SL, Kiens B, Boobis LH, Harman AE: Moderate excersice, postprandial lipemia, and skeletal muscle lipoprotein lipase activity. Metabolism. 2001; 50:756-62.

39. Enevoldsen LH, Simonsen L, Macdonald IA, Bulow J; The combined effects of exercise and food intake on adipose tissue and splanchnic metabolism. J Physiol. 2004; 561: 871-82.

40. Zang JO,Thomas TR,Ball SD:Effect of exercise timing on postprandrial lipemia and HDL colesterol subfractions. J Appl Physiol. 1998; 85:1516-22.

41. Kantor MA, Cullinane EM, Herbert PN, Thompson PD. Acute increase in lipoprotein lipase following prolonged exercise. Metabolism. 1984; 22:454-7.

42. Kantor MA, Cullinane EM, Sady SP, Herbert PN, Thompson $P D$. Exercise acutely increases high density lipoprotein cholesterol and lipoprotein lipase activity in trained and untrained men. Metabolism. 1987; 36:188-92.

43. Van Proeyen K, Szlufcik K, Nielens H, Pelgrim K, Deldicque $L$, Hesselink M, Van Veldhoven $P$, Hespel P. Training in the fasted state improves glucose tolerance during fat - rich diet. J Physiol. 2010; (588)21: 4289 - 4302.

44. Von Duvillard S, Braun W, Markofski M, Beneke R, Leithäuser $R$. Fluids and hydration in prolonged endurance performance. Nutrition. 2004; 20 (7-8): 652-56.

45. De Bock K, Derave W, Eijnde B, Hesselink M, Koninckx E, Rose A, Schrauwen P, Bonen A, Richter E, Hespel P. Effect of trainingin thefasted state on metabolic responses during exercise with carbohydrate intake. J Appl Physiol. 2008; 104:1045-55.

46. Choi YH, Park S, Hockman S, Zmuda-Trzebiatowska $E_{\text {, }}$ Svennelid F, Haluzik M, Gavrilova o, Ahmad F, Pepin L,
Napolitano $M$, et al. alterations in regulation of energy homeostasis in cyclic nucleotide phosphodiesterase 3B-null mice. J Clin Invest. 2006; 116: 3240-51.

47. Schweiger $M$, Schreiber $R$, Haemmerle $G$, Lass $A$, Fledelius $C$, Jacobsen $P$, Tornqvist $H$, Zechner $R$, Zimmermann $R$. Adipose triglyceride lipase and hormone-sensitive lipase are the major enzymes in adipose tissue triacylglycerol catabolism. J Biol Chem. 2006; 281:40236-41.

48. Achten J, Halson SL, Moseley L, Rayson MP, Casey A, Jeukendrup AE. Higher dietary carbohydrate content during intensified running training result in better maintenance of performance and mood state. J Appl Physiol. 2004; 96: 1331-40.

49. Sengene $C$, Bouloumie A, Hauner $H$, Berlan M, Busse $R$, Lafontan M, Galitzky J.Involvement of a CGMP-dependent pathway in the natriuretic peptide-mediated hormonesensitive lipase phosphorylation in human adipocytes. J Biol Chem. 2003;469: 48617-26.

50. Pan DA, Lillioja S, Kriketos AD, Baur LA, Bogardus $C$, Jenkins $A B$. Skeletal muscle triglyceride levels are inversely related to insulin action. Diabetes. 1997; 46:983-8.

51. Goodpaster BH, He J, Watkins S, Kelley DE. Skeletal muscle lipid content and insulin resistance: evidence for a paradox in endurance trained athletes. J Clin Endocrinol Metab. 2001; 86: 5755-61.

52. Bachmann OP, Dahl DB, Brechtel K, Machann J, Haap M, Maier T, Loviscah M, Stumvoll M, Claussen CD, Schick F, Haring $H U$, Jacob S. Effects of intravenous and dietary lipid challenge on intramyocellular lipid content and the relation with insulin sensitivity in humans. Diabetes 2001; 50:2579-84.

53. Schrauwen - Hinderling VB, Kooi ME, Hesselink $M K$, Moonen-Kornips E, Schaart G, Mustard KJ, Hardie DG, Saris WH, Nicolay K, Schrauwen P. Intramyocellular lipid content and molecular adaptations in response to a 1 week high fat diet. Obes Res. 2005; 13:2088-94.

54. Zhang JQ, Thomas TR, Ball SD. Effect of exercise timing on postprandial lipemia and HDL cholesterol subfractions. J Appl Physiol. 1998; 85:1516-22.

55. Moro C, Crampes F, Sengenes C, de Gl, Galitzky J, Thalamas C, Lafontam M, Berlan M. Atrial natriuretic peptide contributes to physiological control of liped mobilization in humans. FASEB 2004b; 18:908-10. 